L'HOMME L'Homme

185-186 | 2008

L'anthropologue et le contemporain : autour de Marc Augé

\title{
Religion et religions
}

En marge du Génie du paganisme

Marcello Massenzio

\section{OpenEdition}

\section{Journals}

Édition électronique

URL : http://journals.openedition.org/lhomme/24184

DOI : $10.4000 /$ lhomme.24184

ISSN : 1953-8103

Éditeur

Éditions de l'EHESS

Édition imprimée

Date de publication : 1 janvier 2008

Pagination : 313-331

ISSN : 0439-4216

Référence électronique

Marcello Massenzio, «Religion et religions », L'Homme [En ligne], 185-186 | 2008, mis en ligne le 01 janvier 2010, consulté le 19 avril 2019. URL : http://journals.openedition.org//homme/24184 ; DOI : 10.4000/lhomme.24184

(c) École des hautes études en sciences sociales 


\section{Religion et religions \\ En marge du Génie du paganisme}

\section{Marcello Massenzio}

3 ÉNIE DU PAGANISME est un ouvrage qui marque un tournant dans le domaine de la réflexion anthropologique sur la religion, la "nôtre » et celle des «autres». Étant donné sa densité, la structure conceptuelle de l'œuvre ne se prête guère à l'exercice du résumé; afin de fournir au lecteur une vision d'ensemble des problèmes traités ainsi que de la méthode élaborée par Marc Augé, il nous a semblé opportun de suivre une approche moins conventionnelle : choisir pour guider notre analyse un passage clé qui concentre l'esprit du livre. Le passage en question concerne deux importants débats : le premier porte sur les caractères comparés du monothéisme et du polythéisme; le second intéresse la pensée occidentale moderne en tant qu'elle est porteuse d'une sensibilité culturelle nouvelle à l'égard de la religion et, en même temps, d'une approche du sacré qui prend ses distances avec les conceptions les plus traditionnelles (sur ce point, Augé établit le dialogue avec le trio composé de Nietzsche, Freud et Bataille). Entre ces deux débats existe un rapport qu'il convient d'expliciter et d'approfondir : nous pensons, en toute hypothèse, qu'en procédant ainsi la portée de l'ouvrage s'impose mieux à l'évidence.

Voici le passage qui lancera notre analyse :

"L'apparent anachronisme des débats actuels sur les mérites comparés du monothéisme et du polythéisme ne doit pas faire illusion: c'est toujours d'une idée de l'homme qu'il y est question et, plus encore, de l'homme concret qui ne s'éveille au sens qu'en société, déjà socialisé, déjà produit par un système qui lui sert à penser et même lui sert de pensée tant qu’il ne lui apparaît pas comme objet possible de pensée » (p. 84). 
Cette phrase nous transporte in medias res: l'anachronisme dont il est question est-il réel ou n'est-il que le produit d'une forme d'inertie intellectuelle? Si notre attention s'arrêtait à la surface des phénomènes hérités des classifications académiques, il faudrait opter pour le premier terme de l'alternative: aujourd'hui, le débat polythéisme/monothéisme ne semble pas susciter un grand intérêt au-delà du cercle restreint des spécialistes, c'est-à-dire des historiens (ou, peut-être, des archéologues) des religions. Or, dès que nous la scrutons en profondeur, cette même question acquiert une importance majeure, ce qui présuppose, en premier lieu, la prise de conscience du rapport de dépendance réciproque existant entre la religion et la société et, en second lieu, la capacité de déchiffrer les modalités concrètes à travers lesquelles les religions polythéistes d'un côté et monothéistes de l'autre, rendent compte de leur fonction sociale. La dualité des dénominations attribuées aux orientations religieuses dissimule, en substance, une diversité nettement plus complexe qui concerne, comme nous pourrons l'observer, le plan de la construction de l'homo socialis; et c'est en référence à ce phénomène, qui ne cesse jamais d'avoir prise sur la conscience des hommes, que Marc Augé interprète la fonction de la religion, comprise en général.

\section{L'individu comme construction religieuse}

Notre analyse met également en jeu deux passages du Génie du paganisme étroitement liés l'un à l'autre :

"L'homme n'aborde jamais les dieux les mains libres et le regard clair. Parler des rapports de l'homme à la religion n'aurait concrètement - psychologiquement et sociologiquement - aucun sens si l'on ne précisait que ces rapports varient avec les cultures et qu'ils varient avec les cultures précisément parce que dans chacune d'entre elles chaque homme est formé par la religion avant d'avoir consciemment avec elle le moindre rapport. Cela, qui nous parait évident dans le cas des sociétés où les visions de l'homme, de la société, du cosmos et des dieux semblent étroitement et solidairement associées aux rituels de naissance, aux systèmes d'éducation et aux initiations, l'est tout autant dans nos sociétés dites laïques pour ceux qui ont eu une "formation religieuse" " (pp. 50-51).

"Ce que nous venons de suggérer à propos des religions païennes polythéistes n'est pas moins vrai, malgré les différences, dans le cas du monothéisme chrétien. L'Église est d'abord un appareil d'éducation et celui-là même qui, adolescent ou adulte, connaît l'épreuve du doute est un homme irrémédiablement chrétien. C'est encore être chrétien que n'être plus croyant» (p. 57).

L'ampleur des thèmes abordés et la richesse de leurs implications théoriques sont évidentes; l'espace restreint d'un article nous oblige à opérer une sélection tout en maintenant l'orientation générale que nous nous sommes fixée. Disons d'emblée notre accord avec Augé pour considérer 
que la fonction éducative - au sens durkheimien du terme ${ }^{1}$ - est le facteur qui, plus que tout autre, caractérise le domaine de la religion. Cette dernière, modelant la pensée de l'individu, œuvre à le rendre conforme au modèle socialement reconnu et, imposant à la pensée l'objet des questions et la façon de les poser, elle prédispose chaque membre du groupe à s'intégrer dans un tissu social spécifique. C'est effectivement la religion qui a la charge fort délicate de gouverner l'appareil devant transmettre l'ensemble des valeurs partagées par la société; elle fournit donc aussi les instruments grâce auxquels chacun confere un sens au monde et à lui-même au moment où il s'ouvre aux relations interpersonnelles. En bref, le renoncement à la "liberté " autonome de la pensée et l'assimilation inconsciente des contraintes imposées par le système représentent les deux visages du processus de socialisation dont la religion est le moteur.

Si cette analyse constitue - dans ses traits essentiels - la toile de fond, nous pouvons commencer à identifier les questions épistémologiques qu'elle soulève. Comment est-il possible de faire de la religion un objet de la pensée étant donné l'importance du conditionnement qu'elle exerce sur la pensée même? En d'autres termes, si l'exercice de toute pensée impose une mise à distance de l'objet, on peut se demander si une telle démarche peut s'appliquer à la religion compte tenu de l'emprise principielle, formatrice, qu'elle exerce sur la pensée. Si nous passons de ce plan général à celui, plus concret, de l'histoire, le problème intéresse plus précisément la civilisation occidentale, qui a introduit le débat théorique sur la religion ; dans un tel cas, la mise à distance suppose que l'on ait acquis un degré suffisant d'autonomie intellectuelle par rapport au christianisme: autonomie par rapport à la manière selon laquelle le christianisme s'impose et s'autoreprésente, pliant à ses propres prérogatives la notion même de religion; autonomie par rapport à la manière selon laquelle le christianisme représente l'univers des religions.

La possibilité d'une position critique sur sa propre religion est la condition nécessaire pour accéder à la compréhension des univers religieux autres, en se tenant à l'écart des idées préconçues et déformantes; ces deux exigences, intimement liées, parcourent la culture occidentale moderne et la caractérisent, focalisant de manière continue l'attention sur le problème - essentiel - de l'eurocentrisme, particulièrement important dans le domaine de la religion, sans doute le plus difficile à contrôler pour les

1. En ce qui concerne cet aspect de la pensée d'Émile Durkheim, le passage suivant nous semble - entre autres - indicatif: «Les représentations religieuses sont des représentations collectives qui expriment des réalités collectives; les rites sont des manières d'agir qui ne prennent naissance qu'au sein des groupes assemblés et qui sont destinés à susciter, à entretenir ou à refaire certains états mentaux de ces groupes" (1994: 13). 
raisons que nous venons d'exposer. Il se trouve que l'exigence de maintenir à distance la forma mentis de matrice chrétienne lorsque la réflexion porte tant sur la notion de religion que sur les religions des autres caractérise la synthèse théorique élaborée par Angelo Brelich dans les Prolégomènes aux volumes de l'encyclopédie de la Pléiade consacrés à l'Histoire des religions (1970: I, 3-59) : c'est pourquoi il nous semble opportun d'esquisser une lecture comparative des perspectives et des solutions théoriques de Brelich et d'Augé.

\section{Deux perspectives de recherche}

Lors de la rédaction des Prolégomènes, Angelo Brelich dut affronter une tâche difficile: il s'agissait de formuler une série de critères scientifiques pour offrir une orientation théorique unitaire aux recherches effectuées par les spécialistes des religions les plus variées, classées selon plusieurs typologies. L'hypothèse la plus simple, au moins en apparence, consistait à élargir la notion courante de religion, de façon à pouvoir l'appliquer aux contextes les plus divers; l'évidence du procédé se heurtait toutefois à une difficulté insurmontable car l'élargissement en question, loin de se réduire à une opération purement quantitative, impliquait (et implique) nécessairement le niveau des contenus. En effet, la notion même de religion qui appartient à la tradition de la pensée occidentale est tellement imprégnée et dépendante de l'esprit du christianisme qu'elle ne peut être exportée, même au prix de transformations et d' "ajustements». De plus, le fait que cette notion exalte la singularité et donc, indirectement, la noncomparabilité de la religion chrétienne, la rendait (et la rend) étrangère au dessein culturel inhérent à l'histoire des religions, fondé sur le postulat de la comparabilité de tous les systèmes religieux.

La solution envisagée par Brelich pour échapper à cette voie sans issue de l'eurocentrisme mérite d'être prise en considération aussi bien pour sa valeur intrinsèque qu'en tant qu'objet de débat. En bref, celle-ci est organisée en deux phases dont la première consiste à élaborer, sur la base des résultats fournis par l'examen comparatif des documents, une sorte d'inventaire des phénomènes évoquant la dimension du religieux, par exemple les mythes, les rites, les croyances en des êtres surhumains, etc. Bien que possédant une connotation spécifique, chacun de ces phénomènes ne déploie pleinement ses potentialités que dans son rapport aux autres, à l'intérieur d'un système de relations réciproques dont la finalité est de médiatiser (ou de garantir) le passage de la nature à la culture. Si l'objectif initial est de construire le "lexique " de la religion - qui représente la plateforme commune des multiples expressions concrètes - le but ultime, 
et c'est la seconde phase du parcours de Brelich, est de rendre raison des différences. Ces dernières dépendent des modalités selon lesquelles chaque formation religieuse a sélectionné ses éléments constitutifs et, en même temps, de ses façons de les mettre en rapport dans une structure unitaire; ajoutons aussitôt que ces modalités et façons ne sont jamais gratuites ou aléatoires mais qu'elles répondent, plus ou moins directement, à des exigences dictées par les systèmes économiques, politiques et sociaux ainsi qu'aux transformations imposées par l'histoire.

Si cette plateforme théorique représente une voie d'accès à la religion en général puisqu'elle possède l'avantage de dépasser le caractère unilatéral de l'approche traditionnelle, elle n'est pourtant pas sans défauts. Brelich fournit, certes, une série de critères qui définissent le dénominateur commun des religions et, par conséquent, permettent de faire apparaître sous un jour nouveau la dimension même de la religion, révélant ainsi une richesse et une plasticité que l'on aurait pu sinon ne pas percevoir. Mais la perplexité vient de la méthode elle-même : celle-ci tend vers un idéal d'objectivité qui impose au chercheur une position au-dessus des partis, équidistante de tous les contextes, que ceux-ci soient proches ou éloignés dans le temps comme dans l'espac. Tout se passe comme si le refus légitime de l'eurocentrisme impliquait aussi que l'on refuse de faire de l'Occident considéré sous l'angle de la religion - un indispensable élément de référence et d'aboutissement de l'analyse.

Il n'est sans doute pas superflu de faire remarquer que c'est la culture occidentale moderne qui a lancé le débat sur la religion, sur son origine, sur sa signification, sur son destin. Cela reflète d'abord la perte d'efficacité progressive des référents institutionnels qui n'ont plus eu la capacité d'imposer la raison d'être du phénomène religieux - que ce soit en général ou relativement au christianisme -, en tant que partie intégrante de l'héritage culturel. Ensuite, ce même débat a donné une impulsion novatrice qui, indirectement ou directement, a stimulé la recherche de nouvelles voies d'accès à la compréhension de la religion; impulsion à laquelle on peut attribuer la formation de l'histoire des religions en tant que discipline dont la méthode comparative appliquée aux diverses religions représente l'axe central.

Dans un tel parcours cognitif, un facteur d'importance fondamentale est l'ouverture aux "religions des autres" (formule conventionnelle qui désigne les formations les plus disparates situées hors de l'orbite du christianisme); cette ouverture requiert une grande prudence méthodologique et la prise de conscience de toutes les difficultés théoriques mises en évidence par Angelo Brelich dans ses Prolégomènes. Autre moment non moins important de ce parcours : l'interaction altérité/identité, la capacité 
d'utiliser l'intelligence de l'autre pour une meilleure compréhension de soi : compréhension des motivations complexes qui sont au fondement de ce débat - passé et présent - sur la religion ; compréhension des caractéristiques spécifiques du christianisme et du processus historique qui les a déterminées.

Si l'on tient compte de cet ensemble d'éléments, la «voie du retour " à l'Occident et celle de son détachement (qui, sur le plan intellectuel, se réalise en mettant ses catégories pour ainsi dire en épochè) se révèlent complémentaires. Considérée sous cet angle, la ligne interprétative de Brelich manifeste un renversement paradoxal : d'un côté elle affronte un des problèmes de fond de la culture occidentale moderne, adoptant une vision des choses et une méthodologie qui reflètent la prise de conscience de la relativité des systèmes de valeurs, de l'autre, elle élude pratiquement les problèmes que pose, au présent, à cette culture, l'existence de la religion, privilégiant la voie du rapport oblique et allusif plutôt que celle du débat ouvert, libre et autonome. On peut se demander si le dépassement de la limite à peine indiquée ne nécessite pas une interpénétration, encore plus approfondie que dans le passé récent, entre l'histoire des religions et l'ethnologie, étant donné que cette discipline s'est posée de manière urgente la question de la relation (et de la nature des relations) entre connaissance de «l'autre» et connaissance de soi $^{2}$.

\section{Monothéisme / polythéisme}

Dans Génie du paganisme, comme dans les Prolégomènes, le concept de religion n'est pas analysé à partir de son élaboration chrétienne. Pour Marc Augé, la conquête d'une autonomie intellectuelle sur les conditionnements du système éducatif est rendue possible par la prise de conscience de leur existence et de leur fonction; à cette fin, le rôle joué par la méthode comparative interculturelle se révèle décisif. Le débat concerne les deux orientations fondamentales qui constituent l'univers des religions, la première dominée par l'idée de transcendance divine et la seconde reposant sur le principe de l'immanence. En vérité, cette distinction ne découle pas d'un examen rigoureux, elle est le produit d'une histoire : il s'agit de la dichotomie imposée par le christianisme pour affirmer sa spécificité et définir son identité, opposant ainsi nettement son credo à celui des autres par une opération qui décrète la séparation du vrai et du faux, de l'absolument positif et de l'absolument négatif dans le domaine du divin. Aussi

2. À cet égard, je me contente de signaler certains ouvrages classiques qui constituent une référence sous le profil théorique: Claude Lévi-Strauss 1955; 1973; Ernesto De Martino 1999a, 2002a: 84-118. 
n'est-ce que dans l'opposition au monothéisme chrétien qu'il est possible de réduire à l'unité le monde des religions qui ne participent pas à la croyance en un Dieu unique et transcendant, laissant ainsi dans l'ombre la diversité de ce monde.

Marc Augé n'a pas pour objet d'introduire (ou de rétablir) les distinctions, de type historique et culturel, au sein des religions de l'immanence; il porte son attention dans une autre direction, par rapport à laquelle la dichotomie, dont nous venons de souligner la source historique, conserve sa raison d'être au-delà de l'approximation qui la caractérise. Le point de départ de son analyse est l'opposition radicale entre monothéisme et polythéisme (dans laquelle le second terme peut être remplacé par celui, plus souple, de paganisme), mais, à peine a-t-il désigné cette opposition qu'il la décompose pour pouvoir la recomposer ensuite à un autre niveau, lui imposant une finalité qui n'a rien de commun avec le projet polémique originaire.

L'antithèse monothéisme/polythéisme se charge d'une signification qui renverse la tradition chrétienne, dans la mesure où la ligne de démarcation entre ces deux termes n'est plus celle qui sépare la vérité du mensonge mais plutôt celle qui passe entre deux formes de "vérité ", deux manières de conférer du sens à une réalité orientée de façons différentes; deux formes qui se prêtent à être interprétées comme les faces opposées d'une réalité unique (la religion), et qui, mises en relation l'une avec l'autre, deviennent intelligibles. Marc Augé transfere, pour ainsi dire, son regard d'ethnologue, habitué à pénétrer le "sens des autres " et à s'interroger sur celui de son propre monde, dans le domaine de l'histoire des religions. Pour ce faire, il choisit une stratégie interprétative complexe, qui fait interagir les données obtenues lors de ses enquêtes ethnographiques conduites en Afrique avec les résultats des analyses de Jean-Pierre Vernant (1965) relatives au polythéisme grec mais aussi, à un autre niveau, avec les réflexions de Marcel Granet (1934) sur le polythéisme chinois - pour ne citer que ses référents les plus connus. Stratégie comparative, seule capable de mettre finalement en pleine lumière le "génie du paganisme».

C'est précisément la confrontation systématique du soi et de l'autre qui, d'une part, ouvre, à nous Occidentaux, la possibilité d'objectiver le système religieux qui nous a construits - elle nous permet aussi bien de le regarder de loin que de l'évaluer par rapport à un point de repère externe et qui, d'autre part, nous donne la possibilité d'étendre les frontières du religieux au-delà des limites que ce système lui-même nous a imposées. Un tel constat nous ramène au problème théorique précédemment souligné mais, avant de refermer le cercle, il est nécessaire de s'arrêter sur quelques thèmes que nous avons jusqu'à présent laissés dans l'ombre. 


\section{La polémique chrétienne}

Un autre passage du Génie du paganisme ouvre et éclaire de nouvelles directions :

«Il reste que l'opposition la plus pertinente qui puisse être faite dans le domaine religieux situerait d'un côté les religions du Dieu unique et personnel, de l'autre les religions de l'immanence, d'un côté les religions missionnaires, de l'autre les polythéismes. L'histoire du monde, qu'on le veuille ou non, est pour une bonne part celle de leur rencontre. Si les polythéismes païens ont toujours perdu [...] c'est, entre autres raisons, à cause de leur exceptionnelle vertu de tolérance" (p. 78).

Un des documents essentiels qui témoigne d'une telle rencontre et qui, en même temps, en fonde le sens dans une perspective chrétienne, est constitué par l'Épître aux Romains de l'apôtre Paul, sur laquelle nous nous attarderons dans le seul but de mettre en lumière ce qui a trait à la polémique religieuse - laquelle présente des accents d'une vigueur remarquable dans son aspect originaire. Sans traiter de problématiques étrangères à notre dessein et qui n'entrent pas dans nos compétences, contentons-nous d'observer que l'Épître en question est un texte d'un extraordinaire intérêt historiographique et anthropologique, qui a fortement contribué à forger la mentalité occidentale et le système de comportements qui la manifeste.

Une partie du premier paragraphe de l'Épître aux Romains est consacrée à éclaircir un problème qui revêt pour son auteur une importance cruciale: l'idolâtrie. Comment a-t-elle pu naître et se répandre dans le monde? Pour comprendre la portée de cette question ainsi que la charge dramatique qui lui est conférée par Paul, on doit expliciter certains liens qui en constituent les prémisses théoriques : le culte des idoles définit l'ensemble des "fausses" religions, celles dont le point central n'est pas constitué par Dieu, le créateur, mais par des êtres qui n'ont point de dignité divine parce qu'ils appartiennent à l'univers des créatures. S'abandonner à l'idolâtrie signifie, avant tout, méconnaître les prérogatives de Dieu : et cela est totalement injustifiable puisque Dieu s'est révélé à tous les hommes, se rendant reconnaissable à travers la création. Dans la pensée de Paul, l'idolâtrie est donc le fruit d'une aberration de l'entendement humain qui, bien que disposant du vrai a préféré le faux :

"Car ce qui, de lui, est invisible, l'éternité de sa puissance et de sa divinité, ses œuvres, depuis la création du monde, l'ont rendu intelligible et par là pleinement visible, afin de rendre les hommes inexcusables.

Connaissant Dieu, ils n’ont pas célébré son éclat, ni ne l'ont remercié, mais ils se sont perdus dans la vanité de leurs calculs et leur cour fermé est entré dans les ténèbres.

Voulant paraître sages, ils sont devenus fous et ils ont échangé l'éclat du Dieu incorruptible contre un semblant d'image d'homme voué à la corruption, ou aussi bien d'oiseaux, de quadrupèdes, de reptiles. 
C'est pourquoi Dieu les a abandonnés à l'impureté, les livrant aux désirs de leurs cœurs, pour déshonorer leurs corps de leur propre fait.

Ceux-là ont échangé la vérité de Dieu contre le mensonge, vénéré et servi la créature en lieu et place du Créateur - qu'il soit béni à travers les âges, amen.

Pour cela, Dieu les a abandonnés à leur passion du déshonneur. Leurs femmes ont substitué à l'usage naturel de leur corps un usage contre nature, de même les hommes, négligeant l'usage naturel de la femme ont brûlé de désir les uns pour les autres, se livrant aux pratiques infamantes des mâles entre eux, recevant en leurs personnes le salaire de leur égarement " (Rom. I, 20-27) ${ }^{3}$.

Dans le thème initial, on pourra sans doute reconnaître une allusion au mythe de la chute, c'est-à-dire au détachement, originaire et coupable, de l'homme et de Dieu qui fonde la condition humaine; la substitution du culte de la vraie divinité par celui des fausses divinités équivaut à un nouveau détachement et à une nouvelle chute. Cette chute est marquée par la régression des hommes, imposée par la colère de Dieu, vers un état chaotique et de pure brutalité, qui se manifeste de façon emblématique dans la sphère de la sexualité, établissant ainsi une continuité entre aberration mentale et aberration sexuelle, problématique à laquelle nous avons réservé ailleurs une analyse (Massenzio 2006).

Quelle est, aux yeux de Paul, la nature du «scandale» implicite de l'idolâtrie ? Ses racines résident dans la distorsion complète de l'essence divine, que l'on fait passer indûment du plan de la transcendance à celui de l'immanence; la violence qui accompagne l'invective de l'Apôtre reflète la condamnation sans appel de l'état de choses produit par une telle déformation et, en même temps, elle est l'expression du zèle dont il fait preuve dans sa tâche pour rétablir la vérité et l'ordre. Au moment même où il prend acte de la fausseté et des erreurs, Paul réaffirme que Dieu, l'éternel, est totalement autre par rapport aux créatures périssables, qu'il est au-delà du créé et du visible, au-delà du temps qui se consume et qui consume, en bref, qu'il est au-delà du plan de l'immanence. "L'ennemi est l'immanence ", pourrions-nous dire en paraphrasant Marc Augé.

Ce passage essentiel de l'Épittre aux Romains synthétise un certain nombre de points destinés à acquérir une importance fondamentale à l'intérieur de l'idéologie chrétienne: d'abord, il place l'histoire humaine sous le signe de l'involution, due au renversement de la vérité contenue dans la révélation divine, à l'origine du temps, selon un schéma de pensée qui persiste dans la théorie moderne de l'Urmonotheismus. Située au cœur même du discours, l'opposition transcendance / immanence traduit un contraste des comportements religieux, dont l'issue sera un choc frontal alimenté par la flamme de l'intolérance. Dans son essence la plus intime, l'idolâtrie exprime une forme 
d'altérité de caractère non primaire mais secondaire, ou "dérivé", en ce qu'elle naît de l'altération de l'identique : elle est en quelque sorte l'identique inversé et, en tant que telle, un monstrueux hybride. Paul prend donc l'engagement de la détruire afin de ramener le genre humain à l'unité affirmée par la révélation; pour Paul, cet engagement représente une mission acceptée au nom du Christ, "par qui nous avons reçu la grâce et la mission d'apôtre, afin que toutes les nations parviennent à l'écoute de la foi et que chez elles son nom grandisse" (Rom. I, 5).

La polémique, dont la lettre de Paul nous offre un témoignage exemplaire, a contribué de manière non marginale à modeler la forma mentis occidentale non seulement en matière de religion mais aussi quant à une certaine façon de concevoir l'histoire, de représenter l' " autre de soi ", d'organiser la relation identité/altérité ; celle-ci est une partie constitutive du christianisme et, par conséquent, de la notion de religion que ce dernier a forgée et véhiculée. À l'intérieur de la civilisation occidentale, l'exigence de remettre en discussion une telle notion, dans la complexité de ses implications, est principalement due au processus de laïcisation de la culture qui a intensifié son propre rythme à partir de la Réforme et de la Renaissance. À cet égard, il nous semble utile d'élargir les limites de notre discours en prenant en compte la pensée d'Ernesto De Martino, historien des religions et ethnologue italien parmi les plus importants du XXe siècle. Il voit dans l'historicisme la méthode permettant d'observer le christianisme avec un recul critique suffisant; d'une part, cette démarche implique de reparcourir le processus de formation et de renforcement du christianisme et, de l'autre, de soumettre à l'analyse les multiples raisons qui ont déterminé la perte d'actualité du message chrétien :

«La perte d'actualité du message chrétien [...] doit être mesurée à l'échelle de la pensée occidentale, à partir du moment où celle-ci se réalise pleinement comme "pensée de l'histoire humaine", c'est-à-dire comme pensée capable d'intégrer dans son rayon d'action la dimension historique dans sa totalité, sans laisser le moindre espace à une intervention surhumaine» (Massenzio $2000: 28$ ).

Perte compensée par l'apparition d'une vision laïque du monde, qui s'affirme parallèlement à la sortie progressive de la religion, au sens proposé par Marcel Gauchet (1985: 133). À la lumière de cette indication, les polémiques engagées par le christianisme - inséparables du système de valeurs qu'il véhicule - sont à considérer non comme des phénomènes se déroulant en dehors de l'histoire (et par conséquent, doués d'une validité absolue) mais comme des produits historiquement conditionnés, articulés à un contexte déterminé, en dehors duquel leur signification originaire s'atténue jusqu'à disparaître, ou bien devient quelque chose de radicalement différent. 


\section{Magie, paganisme, immanence}

Un des livres de De Martino qui aborde au plus près le problème précédemment posé est un classique de l'histoire des religions: Morte e pianto rituale : dal lamento funebre antico al pianto di Maria (2000) ${ }^{4}$. L'ouvrage traite de la rencontre, de la confrontation de deux univers religieux, l'un polythéiste-païen et l'autre chrétien, sur le fond d'une tentative de définition culturelle de la mort humaine, en vue de la résolution de la crise du deuil. À cet égard, la religion chrétienne a diffusé une idéologie qui contraste nettement avec l'orientation dominante des antiques polythéismes de la zone euroméditerranéenne. Ajoutons que le christianisme ne se limite pas à élaborer un système de valeurs propre mais qu'il tend à l'imposer, à lui conférer un statut universel; ce qui l'amène à engager une polémique très dure dont la cible est l'appareil rituel qui reflète la conception polythéiste de la mort qu'il s'agit de délégitimer radicalement.

De Martino situe cette polémique dans une perspective historique - polémique partant des Évangiles pour atteindre son apogée dans les écrits des pères de l'Église - et, en même temps, il la "réécrit " sans parti pris, avec l'intention de faire apparaître le sens païen de la mort, l'opposant dialectiquement à la vision chrétienne du même phénomène. En bref, la voie empruntée par les polythéismes pour conférer une signification culturelle à la mort naturelle part de la reconnaissance de cette dernière en tant que réalité de fait; la religion intervient pour discipliner, pour rendre progressive et acceptable - en vertu du recours au symbolisme mythique la conscience de la mort comme terme ultime et de la distance irrémédiable qui s'interpose entre les morts et les vivants. Le recours à la discipline rituelle, c'est-à-dire aux lamentations funèbres, répond à l'exigence de contenir et de canaliser la crise déclenchée par la force explosive que renferme le deuil, considéré dans son immédiateté.

Le christianisme prend précisément pour cible l'institution des lamentations funèbres en tant qu'elles reflètent une vision de la mort et, plus généralement, du monde, qui apparaît totalement incompatible avec la conception qui repose sur le mythe de la résurrection du Christ, conception qui va au-delà du plan de l'immanence, niant à la fois la réalité de la mort et la séparation irréversible des vivants et des défunts. En contrepartie, le christianisme indique un horizon symbolique qui repose sur l'équivalence de la mort physique et du sommeil, prélude d'un réveil futur. 
Lorsqu'il analyse avec le recul critique de l'historien les positions chrétienne et païenne, Ernesto De Martino se reporte à l'orientation théorique et méthodologique qui inspirait son ouvrage Le Monde magique (1999b) ${ }^{5}$. Son objectif, dans ce livre, est de pénétrer le sens profond de la magie, lui restituant le rang de phénomène culturel de premier ordre qu'elle a perdu après la polémique déclenchée par la pensée philosophique grecque d'abord puis par le christianisme. Nous en tenant à l'essentiel, il ressort une constante de la méthode demartinienne qui historicise l'intolérance du christianisme envers ce qu'il perçoit comme radicalement étranger (qu'il s'agisse de paganisme ou de magie) et qui, en même temps, élabore, par contraste, les critères qui donnent accès à la compréhension des raisons des «autres».

De ce point de vue, le moment le plus intéressant est constitué par son effort pour transformer en méthode de connaissance la prise de conscience de la pluralité des religions et des cultures humaines. L'analyse consacrée au monde magique et la recherche portant sur les lamentations funèbres prennent place dans le cadre d'un nouveau type d'humanisme, que l'auteur qualifie d'" ethnographique» (De Martino 2002b : 395-396) afin de marquer sa distance par rapport à l'humanisme traditionnel, puisque ce qui le caractérise est la place qu'y tient la reconnaissance positive de la diversité. Une telle reconnaissance comporte la mise au point de nouvelles stratégies cognitives visant à un savoir qui soit à la fois savoir de l'autre par rapport à soi et savoir de soi par rapport à l'autre. En bref, la méthodologie demartinienne a pour fondement la dialectique comparative, qui implique la remise en question de la culture occidentale, dans sa multiplicité structurelle, au moment où celle-ci s'ouvre à la connaissance de "mondes " situés au-delà de ses frontières.

Et c'est précisément sur le plan de la méthodologie que se situe le point d'intersection entre la perspective de Ernesto De Martino et celle de Marc Augé, vers lequel il est temps de revenir, en particulier sur quelques problèmes restés sans réponses.

\section{La religion et son objet}

La méthode comparative (au sens que nous lui avons donné) favorise, en général, une compréhension plus large et plus approfondie des phénomènes humains car elle les analyse sur la base de critères qui résultent de

5. Cet ouvrage, paru en 1948, est à l'origine d'un véritable renversement dans la culture italienne, aussi bien dans le domaine de l'ethnologie que celui de l'histoire des religions. La nouveauté de la méthode d'analyse et l'audace des conclusions théoriques ont lancé un grand débat auquel ont participé Benedetto Croce, Mircea Eliade, Raffaele Pettazzoni, Enzo Paci. 
la rencontre de cultures différentes, mises en condition de "dialoguer" entre elles. L'importance de la confrontation interculturelle apparaît encore plus marquée lorsque le projet de connaissance a pour objet la religion, étant donné son pouvoir de conditionnement sur la pensée. Bien que les différents termes du problème aient déjà été illustrés, il faut revenir sur la question pour éclaircir certaines de ses implications.

Définir la notion de religion sans se plier aux contraintes imposées à la pensée par le type de religion en vigueur dans une civilisation déterminée est, disions-nous, une entreprise particulièrement ardue, étant donné que l'individu socialisé a absorbé et intériorisé ces contraintes durant son parcours éducatif. En ce qui concerne la civilisation occidentale, le processus de renouvellement culturel inauguré par l'ethnologie a permis de définir une nouvelle voie d'accès au problème : elle consiste à adopter un point de vue et un critère de mesure du religieux extérieurs aux cadres mentaux de la religion chrétienne. La possibilité d'observer cette dernière avec un certain recul, d'un regard détaché, est la condition nécessaire pour en analyser de manière objective les prérogatives et la dynamique mais, avant tout, elle permet de prendre conscience de sa portée relative, limitée dans l'espace et dans le temps, et donc non absolue. Dans une telle conscience des limites réside, selon nous, le germe de l'attitude critique face à une religion - le christianisme - qui se veut universelle et qui, à l'épreuve des faits, finit par révéler des caractères qui contredisent les schémas mentaux acquis et qui, par conséquent, requièrent d'être interprétés à la lumière de l'histoire.

Cette analyse comparative qui vise à déterminer les spécificités du paganisme par rapport au christianisme (et vice versa) nous offre, en outre, une vision neuve du fait religieux en révélant le fondement humain de la religion, laquelle devient une création culturelle, une pièce centrale du processus de socialisation. Cet élargissement de perspective fait ressortir, par contraste, l'insuffisance de l'approche conventionnelle qui privilégie la composante doctrinale, c'est-à-dire le système de croyances concernant la sphère de l'invisible. S'interroger sur l'objet des religions n'implique pas qu'on détourne notre attention de ce qui est social pour nous concentrer sur la métahistoire ; cela signifie, au contraire, tenter d'explorer la dimension humaine dans un de ses aspects les plus délicats et les plus secrets, situé à la croisée de l'individuel et du collectif.

Aussi, la réflexion de Marc Augé, centrée sur le dieu unique transcendant et sur les divinités immanentes, concerne-t-elle beaucoup moins le divin que l'humain socialisé pour deux types de raisons interdépendantes: d'abord parce que les conceptions antithétiques du divin sont évaluées 
vis-à-vis de la manière de comprendre la réalité et de lui conférer du sens, ensuite parce que la recherche qui s'élabore au fil du Génie du paganisme met principalement l'accent sur le type d'« empreinte » que les religions de l'immanence, comme celles de la transcendance, laissent sur la pensée. À ce point s'ouvre à nous une nouvelle opportunité de créer un lien entre l'orientation théorique de Marc Augé et celle d'Ernesto De Martino. Si le premier entend la religion comme le «lieu» de la construction de l'individu à l'intérieur de la société, le second la présente comme le « lieu » de la construction de la "présence humaine dans le monde ", considérée comme le noyau originaire et, par conséquent, comme la condition sine qua non de l'individu. La "présence ", qu'il faut interpréter à la lumière de la notion heideggerienne de Dasein, se réfere à la qualité qui transforme l'homme en sujet culturel capable de s'opposer à la réalité externe, à la nature.

Nous avons traité ailleurs cette question (Massenzio 1999 : 38-61) et, par conséquent, nous nous limiterons à en proposer ici une synthèse succincte, strictement liée au développement de la présente analyse. Outre son importance primordiale, la présence humaine se signale par sa fragilité intrinsèque, puisqu'elle est constamment exposée au risque de "se perdre ", de se défaire dans des situations qui échappent à la norme; d'où la nécessité d'une refondation continue, obtenue en réitérant, par des rites, le processus mythique qui a assuré sa construction. Rite et mythe évoquent bien sûr la religion et d'après De Martino l'un et l'autre en constituent l'essence ultime. Dans cette perspective l'attention porte non sur la composante doctrinale mais sur le rôle joué par la religion dans la dynamique du passage - qui n'est jamais achevé - de la nature à la culture. La marginalisation de cette composante est l'indice d'une orientation de la pensée qui témoigne de l'existence d'une nouvelle sensibilité culturelle à l'égard de la religion, qui, au-delà des différences qui concernent l'organisation de leurs recherches respectives et les sollicitations intellectuelles auxquelles celles-ci peuvent répondre, rapproche Augé et De Martino.

\section{Les deux débats}

Notre propos se conclura sur un retour au point de départ. Pour récapituler très brièvement nos observations nous retiendrons que la recherche de Marc Augé se déroule sur deux niveaux : le premier concerne le paganisme, objet d'une enquête historique et ethnologique, le second la pensée occidentale confrontée à l'idée de religion en général et, plus précisément, le débat inauguré par Nietzsche, Freud et Bataille ${ }^{6}$ dont les thèses, sous 
des angles différents, donnent la mesure de la complexité et de l'importance de la "question religion" dans la culture occidentale moderne :

« Nietzsche, Freud et Bataille ont quelques traits communs. Tous trois rejettent les religions instituées : Nietzsche rejette à la fois l'idée de religion et l'idée de vérité, Freud considère que la science, contrairement à la religion, n'est pas une illusion, Bataille [...] poursuit une expérience intérieure dont les religions existantes ne peuvent être que les jalons ou les repères. Ce n'est évidemment pas ici le lieu de résumer ou d'analyser leurs œuvres : nous voudrions seulement évoquer ce qu'elles ont de corrosif, de fondamentalement subversif au regard des évidences habituelles (le moi, la société, l'ordre du monde) que l'histoire se charge elle-même de remettre en cause dans les périodes de "crise" : crise de la foi et du sens souvent liées aux difficultés politiques et économiques, ou effervescences religieuses qui ne sont, malgré les apparences, qu'une expression de l'état de crise" (p. 88).

Le lecteur de Génie du paganisme se trouve ainsi en face de deux modèles d'analyse, de deux manières de procéder, de deux façons d'interpréter le même phénomène - la religion - qui pourraient paraître difficilement conciliables, comme s'il s'agissait de langages provenant d'univers éloignés, sans doute trop éloignés pour qu'ils puissent s'harmoniser en dialoguant. C'est du moins l'impression que nous avait laissée cet ouvrage lors d'une première approche. Lorsque nous tentons de remonter à la cause d'une telle "fracture ", il nous semble possible de la repérer essentiellement dans le fait que l'élément commun aux deux débats (que nous définirons de manière approximative, en nommant le premier " anthropologique» et le second "philosophique») est plus apparent que réel en ce que le même terme, "religion ", recouvre de fait des réalités hétérogènes et, par conséquent, se prête à être envisagé à partir de points de vue qui ne sont pas toujours compatibles. Les penseurs modernes à peine cités ont privilégié la recherche abstraite, élaborant des constructions théoriques qui, bien que divergentes (on passe, par exemple, de la religion conçue comme «illusion " à la religion comme recherche d'une originaire intimité perdue) partagent le présupposé qui fait de la religion un phénomène unitaire dans son essence même et donc tendanciellement universel. En ce sens, ces théories, certainement novatrices sur le plan des contenus, obéissent à un pli traditionnel de la pensée européenne. Sur le versant de l'anthropologie, une autre orientation est apparue qui invite à affronter le problème de la religion en partant du concret et du multiple, c'est-à-dire de l'analyse des différents systèmes historiquement donnés pour remonter ensuite, éventuellement, par la comparaison, à la détermination d'une fonction et d'une plateforme théorique unitaire.

Or, une approche plus méditée du Génie du paganisme nous a permis d'apercevoir un plan de raccord entre les deux niveaux de la recherche; 
nous tenterons, pour finir, de l'illustrer en nous appuyant sur un passage de Marc Augé, voisin de celui qui a inauguré notre propos. Se référant au débat sur l'idée de religion, notre auteur relève que le fait de l'ouvrir rend possible de:

«[...] se situer au point limite où des hommes peuvent se croire à l'abri de ce que Freud appelle une "illusion", au point limite où la pensée peut se croire protégée des contraintes qu' exerce sur elle son objet - perspective sans doute elle-même quelque peu illusoire, mais qui reste la seule d'où quelque chose puisse s'entrapercevoir des problèmes que pose aux consciences individuelles (au premier rang desquels il faut mettre celui de leur propre définition) l'existence de la religion» (pp. 84-85).

Cette réflexion mérite d'être soulignée car elle laisse entendre que l'importance des théories formulées par les trois penseurs cités est certes liée à la profondeur de la pensée qui les fonde et aux éclaircissements qui en découlent, mais elle dépend plus encore d'un élément extérieur à la dimension spéculative. En d'autres termes, le débat philosophique acquiert à nos yeux une importance particulière par le fait qu'il atteste de l'exigence intellectuelle, apparue au sein de la civilisation occidentale, de trouver une voie de sortie de la religion, une sorte de zone franche dans laquelle son pouvoir de conditionnement sur la pensée subit un coup d'arrêt, une interruption. Le passage cité du texte de Marc Augé insiste d'ailleurs sur des mots comme "point limite », "protection ", " abri » qui peuvent aussi, dans une certaine mesure, définir l'horizon théorique dans lequel se situe la recherche anthropologique. En effet, il serait impossible de s'interroger sur le "génie du paganisme" sans avoir préalablement cherché (et atteint) le "point limite » où le christianisme relâche - pour le moins - sa prise sur la pensée ; par ailleurs, l'exploration de l'altérité religieuse produit un accroissement du savoir qui, à son tour, favorise l'affranchissement de la pensée des contraintes du système éducatif. C'est dans cette direction qu'il nous semble possible de déterminer un espace commun à partir duquel chacun des deux débats suit, de manière autonome, son propre parcours.

C'est bien connu: la mise en évidence des ressemblances permet la manifestation des divergences; dans notre cas, l'affinité précédemment mise en lumière rend plus nette la ligne de démarcation qui sépare débat anthropologique et débat philosophique. La différence concerne en premier lieu la nature de l'objet d'analyse qui est constitué, d'un côté, par un système d'opposition (polythéisme/monothéisme; immanence/transcendance; tolérance/intolérance) et, de l'autre, par l'idée de religion en tant que telle. Cette dichotomie touche un aspect essentiel de la question, dans la mesure où elle laisse entendre que la conscience de la différence, 
prédominante dans le premier cas, n'a pas d'équivalent dans le second, qui présuppose une conception du phénomène religieux qui transcende des diversités historiques et culturelles. Il s'ensuit que la différence entre les deux débats se prête à être mesurée quant au choix de l'objet et de la méthode de recherche.

Prendre acte des différences historico-culturelles est la condition nécessaire pour procéder à la comparaison, instrument de connaissance au sens où nous l'avons maintes fois défini; en ce qui concerne Génie du paganisme, celle-ci s'incarne dans l'analyse comparée des deux orientations antagonistes: monothéisme et polythéisme, christianisme et paganisme, notre religion et la religion des autres. Cela nous amène à insister sur un point fondamental : l'altérité dans la définition des faits culturels, en général, et des religions, en particulier, constitue le facteur qui plus qu'aucun autre distingue la recherche anthropologique; en mettant en relation dialectique des orientations opposées, celle-ci parvient, par voie déductive, à définir les caractères distinctifs du phénomène religieux (qui, dans l'optique de Marc Augé, renvoie au processus de construction de l'individu).

Le débat sur l'idée de religion, qui se constitue dans le sillon de la tradition spéculative atteint, lui, un résultat contredisant son aspiration à la généralisation; bien que se mouvant sur le plan de l'abstraction théorique, celui-ci approche non tant la substance du phénomène que l'un de ses aspects particuliers, relatif à une période historique précise, mais qui concerne aussi le plan individuel. Dans les termes de Marc Augé, apparaît à la surface "quelque chose [...] des problèmes que pose aux consciences individuelles (au premier rang desquels il faut mettre celui de leur propre définition) l'existence de la religion " (p. 85). Il s'agit de "quelque chose » qui implique cette religion particulière de la transcendance que cristallise la tradition judéo-chrétienne, caractérisée par une certaine manière de modeler l'individu, de concevoir le rapport de l'homme avec Dieu, d'envisager la question de la foi.

Dans le refus de l' «illusion", dans la tendance à libérer la pensée des conditionnements qu'elle implique, on peut apercevoir - disions-nous - la base commune aux deux débats; il nous semble pouvoir affirmer que la recherche anthropologique a effectué un pas en avant dans cette direction, allant au-delà du plan que l'on pourrait appeler «défensif». On doit ce résultat au choix méthodologique de mettre en relation le "soi " et l'"autre de soi » : ce choix se traduit par l'élaboration d'une grille interprétative qui permet non seulement de garder à distance critique la religion mais aussi de transformer en matière à réflexion les contraintes que cette dernière 
impose à la pensée. Considérée dans cette perspective, la médiation de l'altérité constitue, certes, un abri et une protection, mais elle est aussi quelque chose de plus, à savoir la prémisse - non illusoire, probablement - de la maîtrise intellectuelle du phénomène.

Università di Roma-Tor Vergata, Italie massenzio@lettere.uniroma2.it

MOTS CLÉS/KEYWORDS : religion - christianisme/christianity - paganisme/paganism - monothéisme/ montheism - polythéisme/ polytheism. 


\section{Bataille, Georges}

1973 Théorie de la religion. Paris,

Gallimard.

\section{Brelich, Angelo}

1970-1976 « Prolégomènes à une Histoire des religions ", in Henri-Charles Puech, ed., Histoire des religions. Paris, Gallimard

«Encyclopédie de la Pléiade»: I, 3-59.

\section{De Martino, Ernesto}

1999a [1961] La Terre du remords. Le Plessis-Robinson, Institut Synthélabo.

1999b [1948] Le Monde magique. Paris, Institut d'édition Sanofi-Synthélabo.

2000 [1958] Morte e pianto rituale. Dal lamento funebre antico al pianto di Maria. Torino, Bollati Boringhieri.

2002a [1962] Furore Simbolo Valore. Milano, Feltrinelli.

2002b [1977] La Fine del mondo. Contributo all'analisi delle apocalissi culturali. Torino, Einaudi.

\section{Durkheim, Émile}

1994 [1912] Les Formes élémentaires de la vie religieuse. Le système totémique en Australie. Paris, Presses universitaires de France ("Quadrige»).

\section{Freud, Sigmund}

1932 [1927] L'Avenir d'une illusion. Paris, Denoël \& Steele.

1951 [1912-1913] Totem et Tabou. Interprétation par la psychanalyse de la vie sociale des peuples primitifs. Paris, Payot.

\section{Gauchet, Marcel}

1985 Le Désenchantement du monde. Une histoire politique de la religion. Paris, Gallimard.

\section{Granet, Marcel}

1934 La Pensée chinoise. Paris, La

Renaissance du livre.

Lévi-Strauss, Claude

1955 Tristes Tropiques. Paris, Plon.

1973 " Jean-Jaques Rousseau,

fondateur des sciences de l'homme", in Anthropologie structurale deux. Paris, Plon : 45-56.

\section{Massenzio, Marcello}

1999 Sacré et identité ethnique.

Frontières et ordre du monde. Paris, Éd. de l'École des hautes études en sciences sociales

("Cahiers de L'Homme»).

2000 «Religion et sortie de la religion. Le christianisme selon E. De Martino ", Gradhiva 28 : 23-31.

2006 «Il paradosso e la norma. Idolatria e sessualità nelle epistole paoline ", in Olga Pozzi \& Sarantis Thanopulos, eds, Ipotesi gay. Materiali per un confronto. Roma, Borla : 176-195.

\section{Nietzsche, Friedrich}

1970 [1881] Euvres philosophiques complètes, 4. Aurore et Fragments posthumes 1879-1881.

Paris, Gallimard.

1971 [1886-1887] Euvres philosophiques complètes, 7. Par-delà le bien et le mal;

La Généalogie de la morale. Paris, Gallimard.

\section{Vernant, Jean-Pierre}

1965 Mythe et pensée chez les Grecs.

Études de psychologie historique.

Paris, Maspero

(«Les Textes à l'appui»). 